\title{
Evaluating the Burstlet Theory of Inspiratory Rhythm and Pattern Generation
}

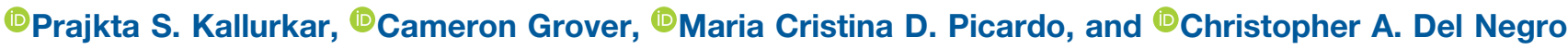

https://doi.org/10.1523/ENEURO.0314-19.2019

Department of Applied Science, Integrated Science Center, Williamsburg, VA 23185

\begin{abstract}
The preBötzinger complex (preBötC) generates the rhythm and rudimentary motor pattern for inspiratory breathing movements. Here, we test "burstlet" theory (Kam et al., 2013a), which posits that low amplitude burstlets, subthreshold from the standpoint of inspiratory bursts, reflect the fundamental oscillator of the preBötC. In turn, a discrete suprathreshold process transforms burstlets into full amplitude inspiratory bursts that drive motor output, measurable via hypoglossal nerve (XII) discharge in vitro. We recap observations by Kam and Feldman in neonatal mouse slice preparations: field recordings from preBötC demonstrate bursts and concurrent XII motor output intermingled with lower amplitude burstlets that do not produce XII motor output. Manipulations of excitability affect the relative prevalence of bursts and burstlets and modulate their frequency. Whole-cell and photonic recordings of preBötC neurons suggest that burstlets involve inconstant subsets of rhythmogenic interneurons. We conclude that discrete rhythm- and pattern-generating mechanisms coexist in the preBötC and that burstlets reflect its fundamental rhythmogenic nature.
\end{abstract}

Key words: breathing; central pattern generator; preBötzinger complex; respiration

\section{Significance Statement}

Breathing movements depend on a neural rhythm and rudimentary motor pattern. Microcircuits of the brainstem preBötzinger complex (preBötC) produce both, but by unknown mechanisms that prove refractory to canonical explanations. Inspired by an unconventional proposal that rhythm and motor pattern are separable processes (Kam et al., 2013a), we replicated their findings that rhythmicity in local preBötC microcircuits can occur independently without obligatory neural bursts that generate motor output. The rhythm is voltage dependent and the constituent interneurons change from cycle to cycle. These results suggest that breathing rhythm is attributable to recurrent excitation among interneurons that discretely trigger neural bursts and motor output. The preBötC, previously considered uniquely rhythmogenic, contains rhythm- and pattern-generating microcircuits.

\section{Introduction}

Breathing is a vital rhythmic behavior. Inspiration, the inexorable active phase of the breathing cycle, originates

Received August 7, 2019; accepted December 17, 2019; First published December 20, 2019.

The authors declare no competing financial interests.

Author contributions: P.S.K., M.C.D.P., and C.A.D.N. designed research; P.S.K. and C.G. performed research; P.S.K., C.G., and C.A.D.N. analyzed data; P.S.K., C.G., and C.A.D.N. wrote the paper.

This work was supported by the National Institutes of Health Grant R01 HL104127 (to C.A.D.N.).

Correspondence should be addressed to Christopher A. Del Negro at cadeln@wm.edu. from the preBötzinger complex (preBötC) in the lower medulla (Smith et al., 1991; Del Negro et al., 2018). preBötC interneurons generate rhythmic activity and project to cranial and spinal premotor and motor neurons that drive inspiratory muscles. However, the neural mechanisms that give rise to inspiratory rhythm and motor pattern are unclear. These mechanisms can be studied in reduced

https://doi.org/10.1523/ENEURO.0314-19.2019 Copyright (C) 2020 Kallurkar et al.

This is an open-access article distributed under the terms of the Creative Commons Attribution 4.0 International license, which permits unrestricted use, distribution and reproduction in any medium provided that the original work is properly attributed. 
preparations that isolate the preBötC and cranial hypoglossal (XII) inspiratory motor circuits, and thus provide an experimentally advantageous minimal breathing-related microcircuit in vitro. Here, we disentangle neural mechanisms intrinsic to the preBötC that engender both inspiratory rhythm and fundamental aspects of the motor pattern.

We stipulate: preBötC bursts propel inspiratory activity to premotor and motor neurons thus they are important for motor pattern. However, are preBötC bursts rhythmogenic? The following observations cast doubt on that premise. The magnitude of inspiratory bursts (in the preBötC and motor output) can be diminished while only minimally affecting their frequency (Johnson et al., 2001; Del Negro et al., 2002; Peña et al., 2004; Pace et al., 2007). Also, manipulating network excitability affects the frequency, but not the magnitude of preBötC inspiratory bursts and motor output (Del Negro et al., 2009; Sun et al., 2019). It thus appears that two discrete phenomena emanate from the preBötC: a fundamental rhythm (whose frequency is adjustable) and a rudimentary pattern consisting of bursts that drive motor output. These appear to be separable processes as codified by Kam and Feldman in their burstlet hypothesis (Kam et al., 2013a; Feldman and Kam, 2015). To describe one cycle, preBötC neurons experience a ramp-like depolarization lasting 100-400 $\mathrm{ms}$, which reflects recurrent excitatory synaptic activity among constituent rhythmogenic neurons (Smith et al., 1990; Rekling et al., 1996; Kam et al., 2013b). This is called the preinspiratory phase because it precedes, and ordinarily leads to, the inspiratory burst. However, Kam and Feldman showed that preinspiratory activity can be divorced from inspiratory bursts by lowering the neural excitability. What often remains is preBötC network activity matching that of the preinspiratory phase but absent the burst; they dubbed these events burstlets (Kam et al., 2013a).

Here, we test the burstlet hypothesis of inspiratory rhythm and pattern generation by explicitly or conceptually repeating Kam et al's (2013a) experiments. We, too, detected preBötC burstlets absent XII output, which were distinct from larger amplitude preBötC bursts accompanied by XII output. Manipulations that lower neural excitability increase the prevalence of burstlets relative to bursts. Composite preBötC rhythm depends on cellular excitability because manipulations of external $\mathrm{K}^{+}$concentration control its frequency. Intracellular recordings and photonic imaging of preBötC inspiratory neurons demonstrate that the burstlets occur in subsets of preBötC neurons, not the entire rhythmogenic population that participates in bursts. Our results also support the fundamental tenet of burstlet theory that preinspiratory activity and burstlets reflect a common rhythmogenic mechanism, and that a threshold process causes burstlets (i.e., preinspiratory activity) to trigger bursts and subsequent motor output. These results imply that pattern generation, although a distinct process from rhythm generation, starts from the preBötC core microcircuit.

\section{Materials and Methods}

The Institutional Animal Care and Use Committee at William \& Mary approved these protocols, which conform to the policies of the Office of Laboratory Animal Welfare (National Institutes of Health) and the guidelines of the National Research Council (National Research Council, 2011). Mice were housed in colony cages on a $14 / 10 \mathrm{~h}$ light/dark cycle with controlled humidity and temperature at $23^{\circ} \mathrm{C}$ and were fed ad libitum on a standard commercial mouse diet (Teklad Global Diets, Envigo) with free access to water.

\section{Mice}

preBötC field recording experiments employed CD-1 mice (Charles River). Whole-cell recordings employed CD-1 mice as well as mice with Cre-dependent expression of fluorescent $\mathrm{Ca}^{2+}$ indicator GCaMP6f dubbed Ai148 by the Allen Institute (RRID:IMSR_JAX:030328; Daigle et al., 2018). We crossed homozygous $D b \times 1^{\text {Cre }}$ (Bielle et al., 2005) females with Ai148 males. We refer to their offspring as Dbx1;Ai148 mice. Newborn Dbx1;Ai148 pups express GCaMP6f in neurons derived from progenitors that express the embryonic transcription factor developing brain homeobox 1 (Dbx1).

\section{Slice preparations}

Neonatal mice (P0-P4) of both sexes were anesthetized by hypothermia and killed by thoracic transection. Brainstems were removed in cold artificial CSF (ACSF) containing the following: $124 \mathrm{mM} \mathrm{NaCl}, 3 \mathrm{mM} \mathrm{KCl}, 1.5 \mathrm{mM} \mathrm{CaCl}_{2}$, $1 \mathrm{mM} \mathrm{MgSO}_{4}, 25 \mathrm{mM} \mathrm{NaHCO}_{3}, 0.5 \mathrm{mM} \mathrm{NaH}_{2} \mathrm{PO}_{4}$, and 30 $\mathrm{mM}$ dextrose, which we aerated with $95 \% \mathrm{O}_{2}$ and $5 \%$ $\mathrm{CO}_{2}$. Brainstems were then glued to an agar block with the rostral side up. We cut a single 450 - to $500-\mu \mathrm{m}$-thick transverse medullary slice with the preBötC at the rostral surface. The position of the preBötC was benchmarked according to neonatal mouse preBötC atlases (Ruangkittisakul et al., 2011, 2014).

\section{Electrophysiology}

Slices were held in place and perfused with ACSF $\left(\sim 28^{\circ} \mathrm{C}\right)$ at $2-4 \mathrm{ml} \mathrm{min}^{-1}$ in a recording chamber on a fixed-stage upright microscope. The external $\mathrm{K}^{+}$concentration, i.e., $\left[\mathrm{K}^{+}\right]_{\mathrm{o}}$, in the $\mathrm{ACSF}$ was initially raised to $9 \mathrm{mM}$, which facilitates robust rhythm and motor output in slices (Funk and Greer, 2013).

Population activity from preBötC interneurons and XII motor neurons was recorded using suction electrodes fabricated from borosilicate glass pipettes (OD: $1.2 \mathrm{~mm}$, ID: $0.68 \mathrm{~mm}$ ). preBötC field recordings were obtained by placing the suction electrode over the rostral face of the preBötC at the surface of the slice. XII motor output was recorded from XII nerve rootlets, which are retained in slices. Signals were amplified by 20,000 , band pass filtered at $0.3-1 \mathrm{kHz}$, and then RMS smoothed using a differential amplifier (Dagan Instruments). Smoothed signals were used for display and quantitative analyses.

We used an EPC-10 patch-clamp amplifier (HEKA Instruments) for whole-cell current-clamp recordings. Patch pipettes were fabricated from borosilicate glass (OD: 1.5 $\mathrm{mm}$, ID: $0.86 \mathrm{~mm}$ ) to have tip resistance of 4-6 M . The patch pipette solution contained the following: $140 \mathrm{mM}$ K-gluconate, $5 \mathrm{mM} \mathrm{NaCl}, 0.1 \mathrm{mM}$ EGTA, $10 \mathrm{mM}$ HEPES, $2 \mathrm{mM} \mathrm{Mg}$-ATP, and $0.3 \mathrm{mM} \mathrm{Na}_{3}$-GTP. We added $50 \mu \mathrm{M}$ 
Alexa Fluor 488 hydrazide dye (A10436, Life Technologies) for visualization after whole-cell dialysis. Whole-cell recordings were made from preBötC inspiratory neurons selected visually based on rhythmic fluorescence changes in Dbx1;Ai148 mice. In CD-1 mice, we only collected wholecell data from preBötC inspiratory neurons that were active in sync with XII motor output. Membrane potential trajectories were low-pass filtered at $1 \mathrm{kHz}$ and digitally recorded at $4 \mathrm{kHz}$ using a PowerLab data acquisition system, which includes a 16-bit analog-to-digital converter and LabChart v7 software (ADInstruments).

We modified $\left[\mathrm{K}^{+}\right]_{\circ}$ in the ACSF from 9 to $3 \mathrm{mM}$ to modulate the network excitability. Each trial consisted of a sequence of non-contiguous $\left[\mathrm{K}^{+}\right]_{\mathrm{o}}$ levels selected randomly in descending order.

Low-amplitude activity in preBötC field recordings was classified as a burstlet if it met these two criteria: the peak of preBötC activity exceeded the mean of the distribution of baseline noise by $2 * S \mathrm{SD}$, and there was negligible concurrent activity in the XII root recording. The mean and SD of baseline noise were obtained by sampling every data point during a sliding 120-s window, constructing a histogram of baseline noise, and fitting that distribution with a Gaussian function to obtain the mean and SD.

A sigh burst in the preBötC field recording was distinguished from an inspiratory burst if it met these three criteria: the area of the putative sigh burst exceeded the mean area of all inspiratory bursts by one SD; the cycle period of the putative sigh bursts measured 1-4 min and not outside this range; and the putative sigh burst was followed by a prolonged inter-event interval $>1.3$ times the average inspiratory cycle time for six consecutive cycles preceding a putative sigh burst (Lieske et al., 2000; Ruangkittisakul et al., 2008; Borrus et al., 2019).

For field recordings, we measured the amplitude (amp) of the preBötC population activity and XII motor output, as well as inspiratory frequency (f; or cycle time). We also measured the rise time, decay time, and duration of burstlets in field recordings. For whole-cell recordings, inspiratory bursts refer to depolarizations with concomitant spiking in preBötC neurons that occur in sync with XII motor output. We measured the amplitude of inspiratory bursts after smoothing to eliminate spikes but preserve the envelope of depolarization. To identify burstlet-like activity in whole-cell recordings, we performed simultaneous field recordings from the contralateral preBötC as well as XII motor output.

We wrote algorithms in MATLAB (RRID:SCR_001622) to calculate the mean frequency as well as the amplitude of bursts and burstlets. The coefficient of variation (CV) of preBötC or XII motor output frequency was calculated as the ratio of $S D$ to the mean frequency.

For all intracellular and two-photon recordings, a neuron that participates in an inspiratory burst is referred as a burst-active neuron and a neuron that participates in a burstlet, is referred as a burstlet-active neuron.

Cycle-triggered averages were calculated and plotted in IgorPro (v.8, RRID:SCR_000325) using the onset of XII output as the event trigger for averaging preBötC inspiratory bursts; the onset of the burstlet itself served as the event trigger for averaging burstlets. We obtained the depolarization rate $(\mathrm{V} / \mathrm{s})$ of the event-triggered averages as the quotient of event amplitude and the elapsed time for that event to reach its peak amplitude. For preinspiratory activity, event amplitude was calculated as the absolute difference between the baseline level of activity and the level of activity in the field recording at the onset of XII motor output. For bursts, event amplitude was calculated as the difference between peak amplitude and field amplitude at the onset of XII motor output (that procedure omits the amplitude portion attributable to the preinspiratory phase). For burstlets, event amplitude was calculated as the difference between peak amplitude and baseline at the onset of the burstlet.

\section{Two-photon imaging}

We imaged intracellular $\mathrm{Ca}^{2+}$ in neurons contained in slices from Dbx1;Ai148 mice using a multi-photon laserscanning microscope (Thorlabs) equipped with a water immersion 20x, 1.0 numerical aperture objective. Illumination was provided by an ultrafast tunable laser with a power output of $1050 \mathrm{~mW}$ at $970 \mathrm{~nm}, 80-\mathrm{MHz}$ pulse frequency, and 100-fs pulse duration (Coherent Chameleon Discovery). We scanned Dbx1;Ai148 mouse slices over the preBötC and collected time series images at 32 $\mathrm{Hz}$. Each frame reflects one-way raster scans with a resolution of $256 \times 256$ pixels $(116 \times 116 \mu \mathrm{m})$. Fluorescence data were collected using Thorlabs LS software and then analyzed using Fiji (Schindelin et al., 2012; Schneider et al., 2012), MATLAB, and IgorPro.

Regions of interest (ROIs) representing individual $D b \times 1$ derived preBötC neurons were detected using MATLAB. First we found the set of collective inspiratory bursts in the time series from each Dbx1;Ai148 slice by averaging the fluorescence intensity of all pixels for each frame; fluorescence peaks are easily detectable periodic events. The collection of cycle periods is normally distributed; the $95 \%$ confidence intervals (Cls) are defined by the mean cycle period $\pm 2 * \mathrm{SD}$.

Next, we down-sampled the planar resolution of our stack of images by $2^{n}(n \geq 1)$. The mean fluorescence of the constituent pixels was assigned to each composite pixel. We performed temporal fast Fourier transforms (FFTs) on the composite pixels. The maximum FFT value within the 95\% Cls for slice frequency (determined above) was then mapped to a corresponding position in a new processed two-dimensional image. This method quantifies how strongly a composite pixel changes fluorescence at frequencies that correspond to inspiratory rhythm. After having created the complete processed image, we computed the mean and SD for FFT values associated with all composite pixels. Any composite pixel with intensity less than mean $+2 * S D$ was set to zero. Any contiguous remaining pixel sets (whose diameter exceeds $6 \mu \mathrm{m}$ ) were retained as ROls.

We then applied the set of ROls to analyze $\mathrm{Ca}^{2+}$ transients in the original fluorescence imaging stack using the equation $\left(F_{i}-F_{0}\right) / F_{0}$, i.e., $\Delta F / F_{0}$, where $F_{i}$ is the instantaneous average fluorescence intensity of all the pixels in a given $\mathrm{ROI}$ and $\mathrm{F}_{0}$ is the average fluorescence intensity of all the pixels within the same $\mathrm{ROI}$ averaged over the entire time series. Finally, we smoothed the $\Delta F / F_{0}$ time series 
with a forward moving average with a window of four time points.

\section{Statistics}

All the null hypothesis statistical tests were calculated using Prism (v.8, RRID:SCR_002798). Changes in the frequency and amplitude of preBötC field activity and XII output as a function of $\left[\mathrm{K}^{+}\right]_{\text {o }}$ were evaluated using linear regression. Changes in the rise time, decay time, and duration of burstlets were evaluated using linear regression. We compared group means using either Student's paired $t$ test or repeated measures one-way ANOVA, applying Holm-Sidak's multiple comparison test post hoc. We compared the variability of frequency of preBötC events and XII motor output using a Kruskal-Wallis test, applying Dunn's multiple comparison test post hoc. We compared the frequency of preBötC events using Welch's $t$ test.

\section{Results}

preBötC generates bursts at high levels of excitability but burstlets appear as excitability decreases

We manipulated excitability by varying $\left[\mathrm{K}^{+}\right]_{0}$ by integer units between 9 and $3 \mathrm{mM}$. At high $\left[\mathrm{K}^{+}\right]_{\text {o }}$ (e.g., 9 or $7 \mathrm{mM}$ in Fig. $1 A$ ), we observed mostly burst events, defined by peaks of activity in the preBötC field recording with coincident XII output. Nevertheless, we also observed events in the preBötC field recording whose amplitudes measured $15-65 \%$ of the bursts and occurred without coincident XII discharge (Fig. 1A).

We detected and then studied low-amplitude preBötC events whose peaks exceeded the $95 \% \mathrm{Cls}$ of baseline noise (Fig. $1 A$, insets). That detection process ensures that low-amplitude events are unlikely (with probability $<0.05$ ) to be ordinary uncoordinated fluctuations of neural activity. The alternative is that these low-amplitude preBötC events reflect coherent network activity.

If the low-amplitude events reflect burstlets as defined by Kam and Feldman (Kam et al., 2013a), then they should be more abundant at low levels of excitability where the collective activity of rhythmogenic neurons may not reach the threshold for burst generation. Visual inspection of the traces in Figure $1 A$ shows that to be the case, i.e., lowamplitude events devoid of XII output are more abundant at 5 and $3 \mathrm{mM}\left[\mathrm{K}^{+}\right]_{\circ}$ compared to 9 and $7 \mathrm{mM}\left[\mathrm{K}^{+}\right]_{\text {。 }}$.

At $3 \mathrm{mM}\left[\mathrm{K}^{+}\right]_{0}, 70 \pm 3 \%$ ( $n=12$ slices) of detected preBötC events occurred without concomitant XII output. We quantified the relative abundance of low-amplitude versus burst events for the entire data set (Fig. 1B, $n=19$ slices). At incrementally higher $\left[\mathrm{K}^{+}\right]_{0}$ levels, the relative fraction of low-amplitude events decreases in a sigmoidal fashion such that they comprise only $5.2 \pm 6.3 \%(n=19$ slices) of the preBötC events at $9 \mathrm{mM}$. We conclude that low-amplitude preBötC events, absent motor output, reflect burstlets as defined previously (Kam et al., 2013a).

\section{Burst-burstlet and sigh rhythmic frequencies vary as a function of network excitability}

We measured the frequency of preBötC rhythm ( $f_{\text {preBötc }}$, which we refer to as composite rhythm because the

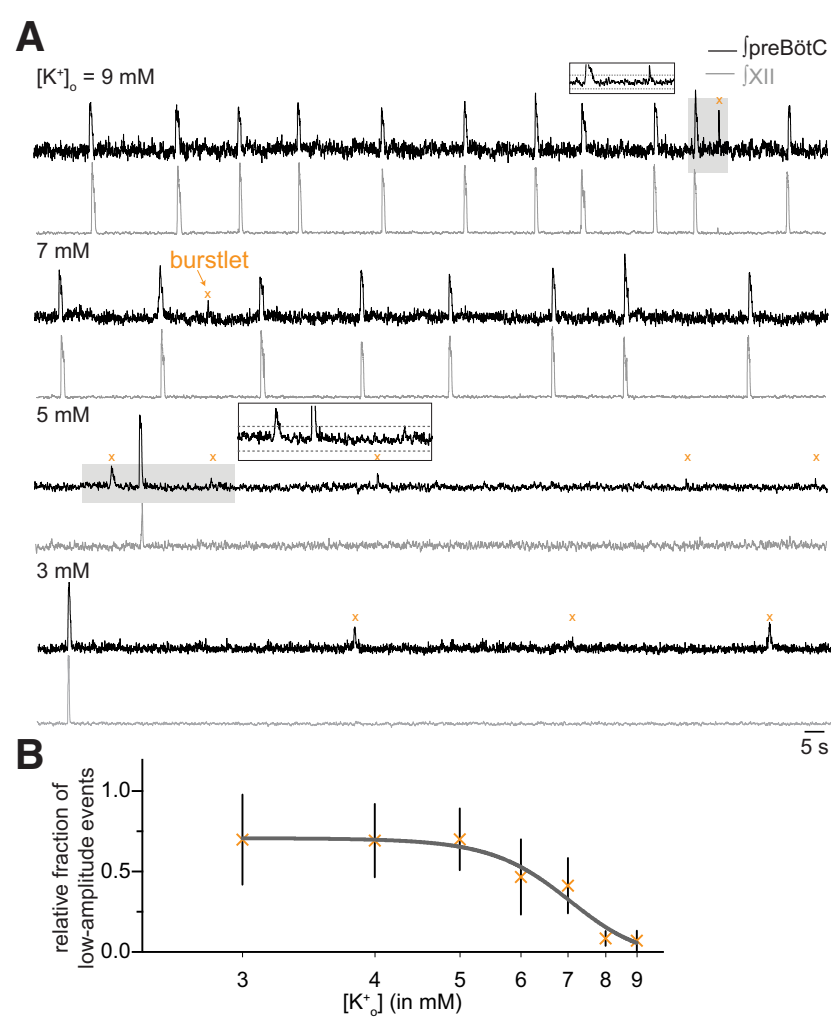

Figure 1. preBötC field and XII recordings demonstrate inspiratory burst and "burstlet" rhythms. A, preBötC field (black) and XII (gray) recordings at different levels of $\left[\mathrm{K}^{+}\right]_{0}$. Insets show expanded traces from gray boxes. Dashed lines in the insets mark the $95 \%$ Cls. Orange $\times$ symbols indicate burstlets. Time calibration applies to all traces. $\boldsymbol{B}$, The relative fraction of burstlets in preBötC field recordings as a function of $\left[\mathrm{K}^{+}\right]_{0}$. Vertical bars show SD.

measured events consist of either bursts or burstlets) and XII motor output $\left(\mathrm{f}_{\mathrm{XII}}\right)$ at different $\left[\mathrm{K}^{+}\right]_{\mathrm{O}}$ levels (Fig. $2 A)$ similar to Kam et al. (2013a). They measured the $\mathrm{f}_{\text {preBötc }}$ and $\mathrm{f}_{\mathrm{XII}}$ at three discrete $\left[\mathrm{K}^{+}\right]_{\mathrm{o}}$ levels $(3,6$, and 9 $\mathrm{mM}$ ) and reported significantly lower $\mathrm{f}_{\mathrm{XII}}$ at $3 \mathrm{mM}$ compared to either 6 or $9 \mathrm{mM}$. Regarding $f_{\text {preBötc }}$, it was lower at $3 \mathrm{mM}$, yet there was no difference between the $\mathrm{f}_{\text {preBötc }}$ measured at 6 vs $9 \mathrm{mM}$ (Kam et al., 2013a, see their Fig. $1 A$ and Table 1). Those data do not resolve the relationship between composite rhythm and network excitability so we measured rhythmic activity at all integer $\left[\mathrm{K}^{+}\right]_{\mathrm{o}}$ levels between 3 and $9 \mathrm{mM}$. $\mathrm{f}_{\text {preBötc }}$ and $\mathrm{f}_{\mathrm{XII}}$ increased linearly as the excitability increased (in this report: Fig. $2 A$, Table 1, which reports linear regression tests). Additionally, $f_{\text {preBötc }}$ and $f_{X I I}$ differed significantly at both 3 and $4 \mathrm{mM}\left[\mathrm{K}^{+}\right]_{\mathrm{o}}$ (Welch's $t$ test, $p=0.009$ and $p=0.031$, respectively) which maps to the portion of the curve in Figure 1B, where the relative fraction of burstlets plateaus at $70 \pm 30 \%(n=12$ slices $)$ and thus explains the relative sparsity of XII events compared to preBötC burstlets. Manipulations of network excitability influence the frequency of composite rhythm and the relative fraction of burstlets that comprise it.

Changes in the network excitability affect the periodic variability of XII motor output (Del Negro et al., 2009). We 
A
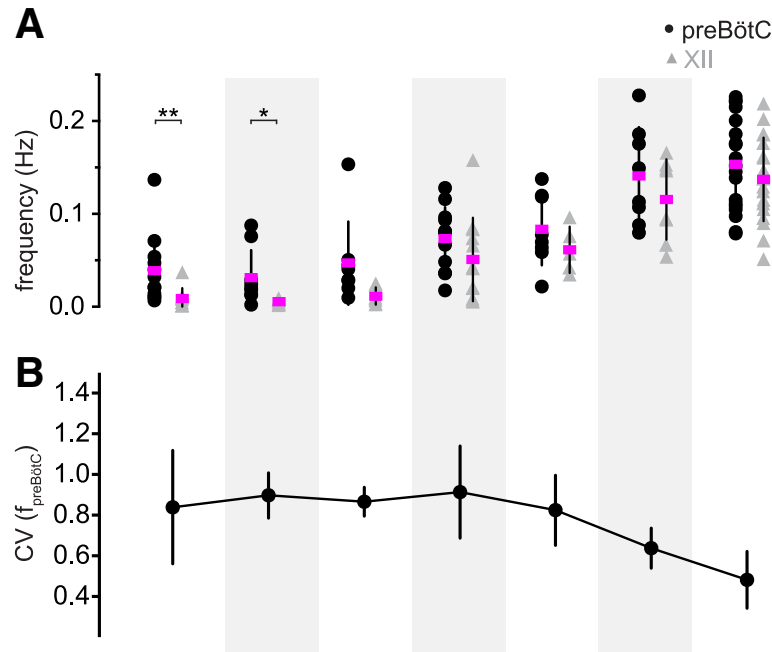

C
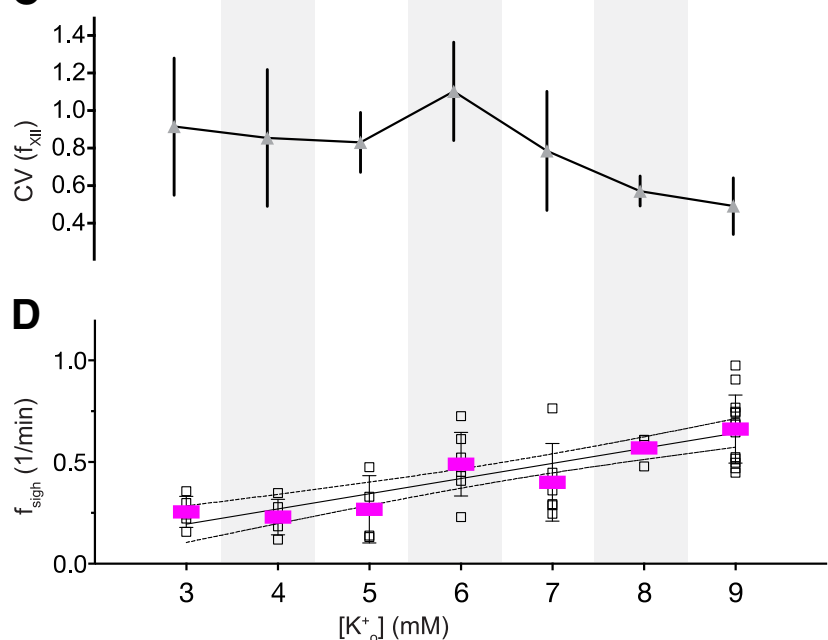

Figure 2. Frequency and variability of preBötC composite rhythm, XII motor output, and sigh rhythm. $\boldsymbol{A}$, Frequency of composite rhythm ( $f_{\text {preBötc }}$, filled circles) and XII motor output $\left(f_{\mathrm{XII}}\right.$, gray triangles) as a function of external $\mathrm{K}^{+}$concentration, i.e., $\left[\mathrm{K}^{+}\right]_{\mathrm{O}}$. The frequency from each slice preparation is shown with mean frequency (magenta) and SD (vertical lines). Statistical significance is indicated by symbols as $* p<0.05$, $* * p<0.01$. $\boldsymbol{B}$, C, Mean CV for composite (filled circles) and XII (gray triangles) rhythms as a function of $\left[\mathrm{K}^{+}\right]_{0}$. Vertical bars show SD. $\boldsymbol{D}$, Frequency of sigh rhythm $\left(f_{\text {sigh }}\right.$, black unfilled squares). The frequency from each slice preparation is shown with mean frequency (magenta) and SD (vertical lines). Light gray background shading was applied to differentiate $\left[\mathrm{K}^{+}\right]_{\mathrm{o}}$ levels.
Table 2. Effects of $\left[\mathrm{K}^{+}\right]_{0}$ on the rise time, decay time, and duration of burstlet

\begin{tabular}{|c|c|c|c|}
\hline$\left[\mathrm{K}^{+}\right]_{\mathrm{o}}(\mathrm{mM})$ & Rise time (ms) & Decay time (ms) & Duration (ms) \\
\hline 3 & $124.9 \pm 70.5$ & $317.9 \pm 184.8$ & $400.1 \pm 165.4$ \\
\hline 4 & $173.1 \pm 97.4$ & $262.1 \pm 164.1$ & $394.1 \pm 220.8$ \\
\hline 5 & $130.7 \pm 79.5$ & $236.6 \pm 83.5$ & $375.2 \pm 163.2$ \\
\hline 6 & $208.4 \pm 105.3$ & $232.8 \pm 92.9$ & $352.7 \pm 129.2$ \\
\hline 7 & $173.9 \pm 39.9$ & $246 \pm 89.9$ & $350.2 \pm 94.8$ \\
\hline 8 & $135.3 \pm 59.7$ & $255.2 \pm 125.8$ & $361.4 \pm 132.3$ \\
\hline 9 & $155.6 \pm 77.8$ & $198.2 \pm 66.4$ & $310.3 \pm 86$ \\
\hline \multicolumn{4}{|c|}{ Linear regression test } \\
\hline Slope $(\mathrm{ms} / \mathrm{mM})$ & 1.25 & -13.75 & -13.60 \\
\hline$p$ value & 0.067 & 3.199 & 2.245 \\
\hline$R^{2}$ & 0.001 & 0.060 & 0.043 \\
\hline
\end{tabular}

The rise time, decay time, and duration of burstlet are reported as mean \pm $\mathrm{SD}$. All the results were analyzed using linear regression analysis. Number of slices $(n)$ at each $\left[\mathrm{K}^{+}\right]_{\mathrm{O}}(\mathrm{mM}, n)$ : $(3,8) ;(4,9) ;(5,6) ;(6,8) ;(7,8) ;(8,8) ;(9$, 14)

reexamined that principle and further tested the variability of the composite preBötC rhythm (Fig. $2 B$ ). The variability of $\mathrm{f}_{\mathrm{XI}}$, quantified by $\mathrm{CV}$, peaked at $\sim 1.1$ when $\left[\mathrm{K}^{+}\right]_{\text {o }}$ was 6 $\mathrm{mM}$ (Fig. 2C; Table 3). In contrast, the $\mathrm{CV}$ of $\mathrm{f}_{\text {preBötc }}$ remained between 0.9 and 0.7 over low to medium levels of excitability (3-6 $\mathrm{mM}\left[\mathrm{K}^{+}\right]$) without peaking at $6 \mathrm{mM}$ $\left[\mathrm{K}^{+}\right]_{\mathrm{o}}$ (Fig. 2B; Table 3). These data suggest that peak CV of $\mathrm{f}_{\mathrm{XII}}$ at $6 \mathrm{mM}\left[\mathrm{K}^{+}\right]_{0}$, an intermediate level of excitability, is not attributable to instability in the preBötC rhythm. It rather reflects the equal probability of evoking either burstlets (absent XII output) and bursts with XII output (Fig. $1 B$ or Kam et al., 2013a, their Fig. 2), which makes the periodic XII output more variable than preBötC activity.

The variability of $f_{\text {preBötc }}$ and $f_{X \mid I}$ are lowest at $9 \mathrm{mM}$ $\left[\mathrm{K}^{+}\right]_{\circ}$ (Fig. 2B,C; Table 3). ACSF containing $9 \mathrm{mM}\left[\mathrm{K}^{+}\right]_{\circ}$ represents the empirically determined ideal conditions for rhythmically active slices (Funk and Greer, 2013; Smith et al., 1991) where the likelihood of burstlets is minimal (Fig. 1B; Kam et al., 2013a, their Table 1).

We also examined the frequency of the sigh rhythm $\left(f_{\text {sigh }}\right)$, which increased linearly as the network excitability increased by means of $\left[\mathrm{K}^{+}\right]_{\mathrm{o}}$ (Fig. 2D; Table 1).

We also examined the amplitude of bursts, burstlets, and XII output. The amplitude of preBötC bursts and XII output were invariable over all $\left[\mathrm{K}^{+}\right]_{\text {。 }}$ levels (Fig. $3 A, C$ ), confirmed using linear regression tests (Table 1). However, the burstlet amplitude increased from $1.2 \pm 0.6 \mathrm{mV}$

Table 1. Linear regression analyses of the effects of $\left[\mathrm{K}^{+}\right]_{\mathrm{o}}$ on the frequency and amplitude of preBötC events and XII motor output

\begin{tabular}{|c|c|c|c|c|}
\hline & $\begin{array}{c}\text { Slope } \\
(\mathrm{Hz} / \mathrm{mM} \text { or } 1 / \mathrm{min} \mathrm{mM} \text { or } \mathrm{V} / \mathrm{mM})\end{array}$ & $p$ value & $R^{2}$ & Figure \\
\hline preBötC composite frequency $(\mathrm{Hz})$ & 0.021 & $3.0 \mathrm{E}-14$ & 0.55 & $2 A$ \\
\hline XII motor output frequency $(\mathrm{Hz})$ & 0.024 & $2.0 \mathrm{E}-17$ & 0.68 & $2 A$ \\
\hline Sigh frequency $(1 / \mathrm{min})$ & 0.074 & $1.9 \mathrm{E}-8$ & 0.53 & $2 D$ \\
\hline Burst amplitude (V) & 0.049 & 0.673 & 0.002 & $3 A$ \\
\hline Burstlet amplitude (V) & 0.179 & 0.0001 & 0.19 & $3 B$ \\
\hline XII amplitude (V) & 0.148 & 0.497 & 0.01 & $3 C$ \\
\hline
\end{tabular}

$\left[\mathrm{K}^{+}\right]_{0}$ was changed from 3 to $9 \mathrm{mM}$ in $1 \mathrm{mM}$ steps. All results were analyzed using linear regression analysis. Number of slices $(n)$ at each $\left[\mathrm{K}^{+}\right]_{0}(\mathrm{mM}, n)$ : $(3$, $12) ;(4,9) ;(5,8) ;(6,12) ;(7,8) ;(8,8) ;(9,19)$. 
Table 3. Effects of $\left[\mathrm{K}^{+}\right]_{\mathrm{o}}$ on the variability of frequency of preBötC composite rhythm and XII motor output

\begin{tabular}{llll}
\hline & $\mathrm{H}(\mathrm{df})$ & $p$ value & \multicolumn{1}{c}{ Post hoc analysis } \\
$\mathrm{CV}\left(\mathrm{f}_{\text {preBötc }}\right)$ & $\mathrm{H}(7)=19.61$ & 0.003 & 6 vs $9 \mathrm{mM}\left[\mathrm{K}^{+}\right]_{\mathrm{O}}: 0.005$ \\
$\mathrm{CV}\left(\mathrm{f}_{\mathrm{XII}}\right)$ & $\mathrm{H}(7)=29.01$ & $1.408 \mathrm{E}-5$ & 6 vs $8 \mathrm{mM}\left[\mathrm{K}^{+}\right]_{0}: 0.039$ \\
& & 6 vs $9 \mathrm{mM}\left[\mathrm{K}^{+}\right]_{\mathrm{O}}: 7.968 \mathrm{E}-7$ & $2 A$ \\
& & & $2 B$ \\
\hline
\end{tabular}

The reported Kruskal-Wallis test statistic $(\mathrm{H})$, degrees of freedom (df), and $p$ values compare frequency variability of composite rhythm and XII motor output as $\left[\mathrm{K}^{+}\right]_{\mathrm{o}}$ is changed from 3 to $9 \mathrm{mM}$. Post hoc analysis (calculated using Dunn's test) report $p$ values for the group that were statistically significant.

at $3 \mathrm{mM}\left[\mathrm{K}^{+}\right]_{\mathrm{o}}(n=12$ slices $)$ to $2.3 \pm 1.0 \mathrm{mV}(n=13$ slices) at $9 \mathrm{mM} \mathrm{K}^{+}$(Fig. 3B), which linear regression showed was unlikely to occur by chance if the slope of burstlet amplitude versus $\left[\mathrm{K}^{+}\right]_{0}$ was actually zero $(p=$ 0.001 ; Table 1). These results show that burstlet amplitude depends on network excitability whereas preBötC burst and XII motor output amplitudes do not.

We measured the rise time, decay time, and duration of burstlets, but there were no trends across $\left[\mathrm{K}^{+}\right]_{\mathrm{o}}$ levels, confirmed using linear regression (Table 2).

\section{Burstlets evolve bilaterally and underlie the pre- inspiratory phase of preBötC bursts}

If burstlets reflect coherent preBötC rhythmicity, then they should be bilaterally synchronous. To test that prediction, we recorded preBötC activity from both sides of

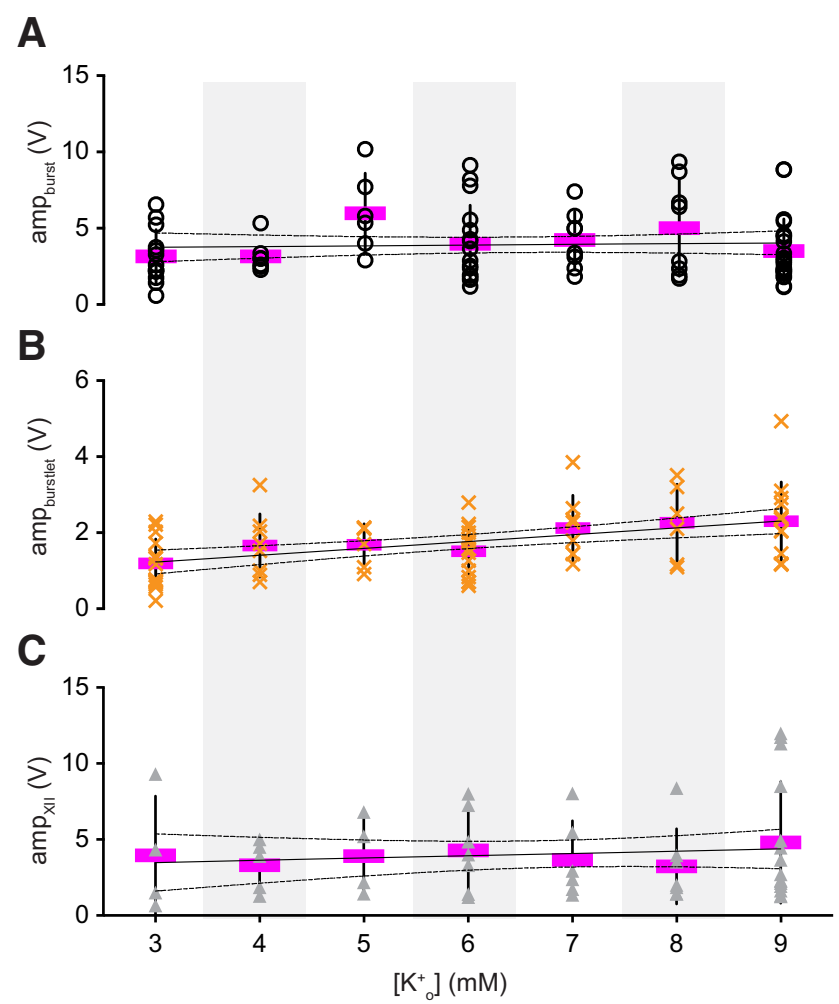

Figure 3. Amplitude of preBötC events and motor output. $\boldsymbol{A}-\boldsymbol{C}$, Amplitude of inspiratory bursts (amp burst, open circles), burstlets

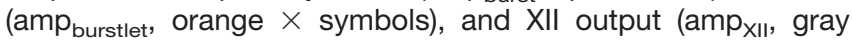
triangles) as a function of external $\mathrm{K}^{+}$concentration, i.e., $\left[\mathrm{K}^{+}\right]_{0}$. The amplitude of each slice preparation is shown with mean amplitude (magenta) and SD (vertical lines). Linear regression lines (solid) and 95\% Cls (dashed) are shown. Light gray background shading was applied to differentiate $\left[\mathrm{K}^{+}\right]_{\mathrm{o}}$ levels. slices along with XII motor output (Fig. 4A); $97 \pm 4 \%$ and $92 \pm 7 \%$ of preBötC burstlets were bilaterally synchronous at 6 and $3 \mathrm{mM}\left[\mathrm{K}^{+}\right]_{0}$, respectively ( $n=4$ slices). The bilateral preBötC bursts commence $\sim 400$ ms before the onset of XII motor output (Fig. 4A, inset), which is considered the preinspiratory phase and the hallmark of rhythm generation (Smith et al., 1990; Rekling et al., 1996; Kam et al., 2013a; Feldman and Kam, 2015; Del Negro et al., 2018).

If burstlets reflect the preinspiratory component of inspiratory bursts, as proposed by Kam and Feldman, then their trajectories should look alike when superimposed. At $6 \mathrm{mM}\left[\mathrm{K}^{+}\right]_{0}$, the rising phase of the burstlet resembles the preinspiratory phase of the inspiratory burst (Fig. 4B). We compared the depolarization rates of the rising phase of

\section{A}

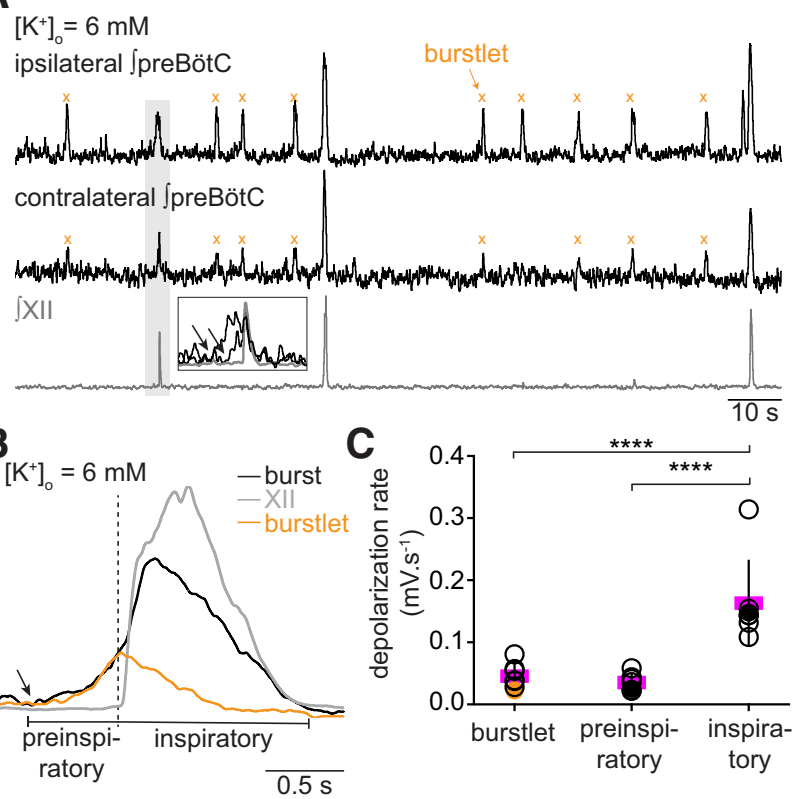

Figure 4. Burstlets are bilaterally synchronous and makeup the preinspiratory phase of bursts. $\boldsymbol{A}$, Bilateral field recordings of the preBötC, with XII output (gray). B, Cycle-triggered averages of burstlets (orange), inspiratory bursts (black), and XII output (gray). The onset of preinspiratory preBötC activity and burstlets are marked by an arrow; their peaks occur at the onset of XII motor output, marked by vertical dashed line. For inspiratory bursts, the onset of XII motor output marks the onset of the event. $\boldsymbol{C}$, Plot of rising slope of burstlets, the preinspiratory phase of bursts, and the inspiratory burst itself. The depolarization rate for each slice is shown with individual symbols (filled symbols are shown in the example in $\boldsymbol{B}$ ); the mean shown in magenta. Asterisks indicate statistical significance at $p<$ 0.0001 . 
burstlets, the preinspiratory phase of inspiratory bursts, and the rising phase of inspiratory bursts. The rising phase of burstlets is comparable to the rising phase of the preinspiratory activity, but the rising phase of both burstlets and preinspiratory activity are incommensurate with the rising phase of inspiratory bursts (one-way ANOVA, $F_{(2,12)}=33.76, p=1.2 \mathrm{E}-5$; burstlet vs preinspiratory, $p=$ 0.577 ; burstlet vs inspiratory, $p=2.5 \mathrm{E}-5$; and preinspiratory vs inspiratory, $p=3.7 \mathrm{E}-5 ; n=7$ slices; Fig. $4 C$ ). These data suggest that burstlets and the preinspiratory phase of bursts reflect the same underlying process, which is distinct from the process underlying full inspiratory bursts.

\section{Burstlets are the summation of EPSPs in preBötC neurons}

We examined how individual preBötC inspiratory neurons contribute to collective events detected in field recordings (bursts and burstlets) via whole-cell recordings in CD-1 $(n=7)$ and Dbx1;Ai148 mouse $(n=3)$ slices. Dbx1;Ai148 mouse pups express genetically encoded $\mathrm{Ca}^{2+}$ reporter GCaMP6f in Dbx1-derived preBötC neurons obligatory for breathing rhythmogenesis (Bouvier et al., 2010; Gray et al., 2010; Wang et al., 2014; Cui et al., 2016; Vann et al., 2016, 2018; Baertsch et al., 2018).

In control conditions $\left(9 \mathrm{mM}\left[\mathrm{K}^{+}\right]_{\mathrm{o}}\right)$, inspiratory drive potentials synchronized with XII motor output during almost all cycles (96 $\pm 7 \%, n=16$ preBötC neurons recorded in slices from 10 different animals). We considered those events bursts. We then modified the excitability by changing $\left[\mathrm{K}^{+}\right]_{\circ}$ to either $6 \mathrm{mM}(n=11$ neurons in eight slices) or $7 \mathrm{mM}$ ( $n=6$ neurons in three slices). We recorded drive potentials of 6- to $10-\mathrm{mV}$ amplitude that were not accompanied by XII motor output. We considered those events burstlets (Fig. 5A).

During bursts, preBötC neurons, from a baseline membrane potential of $-60 \mathrm{mV}$ (maintained below the activation threshold of persistent $\mathrm{Na}^{+}$current via bias current), exhibit inspiratory drive potentials exceeding $20 \mathrm{mV}$ and intraburst spiking of 2-17 spikes/burst $(\sim 6-60 \mathrm{~Hz})$. During burstlets, these same neurons exhibit EPSPs that summate during the burstlets (Fig. $5 A$, insets) as well as spikes (Fig. $5 B$, cycle-triggered average from a whole-cell recording). We never observed a preBötC neuron ( $n=$ $0 / 17$ neurons in 10 slices) that was active during burstlets but not bursts.

Burstlets resemble the preinspiratory phase of bursts. This applies to field recordings (Fig. $4 B$ ) and whole-cell recordings at both $\left[\mathrm{K}^{+}\right]_{\mathrm{o}}$ levels: $7 \mathrm{mM}(n=4$ out of six neurons) and $6 \mathrm{mM}$ ( $n=6$ out of 11 neurons; Fig. 5B). These are the first intracellular recordings to show that burstlets reflect the temporal summation of EPSPs, often crossing threshold to generate repetitive spiking, in preBötC neurons.

\section{preBötC inspiratory neurons do not participate in every burstlet}

Kam et al. (2013a) showed that $89 \%$ of the inspiratory preBötC neurons take part in burstlets. We retested that notion by comparing $f_{\text {preBötc }}$ and $f_{X I I}$ monitored during separate or simultaneous whole-cell and field recordings
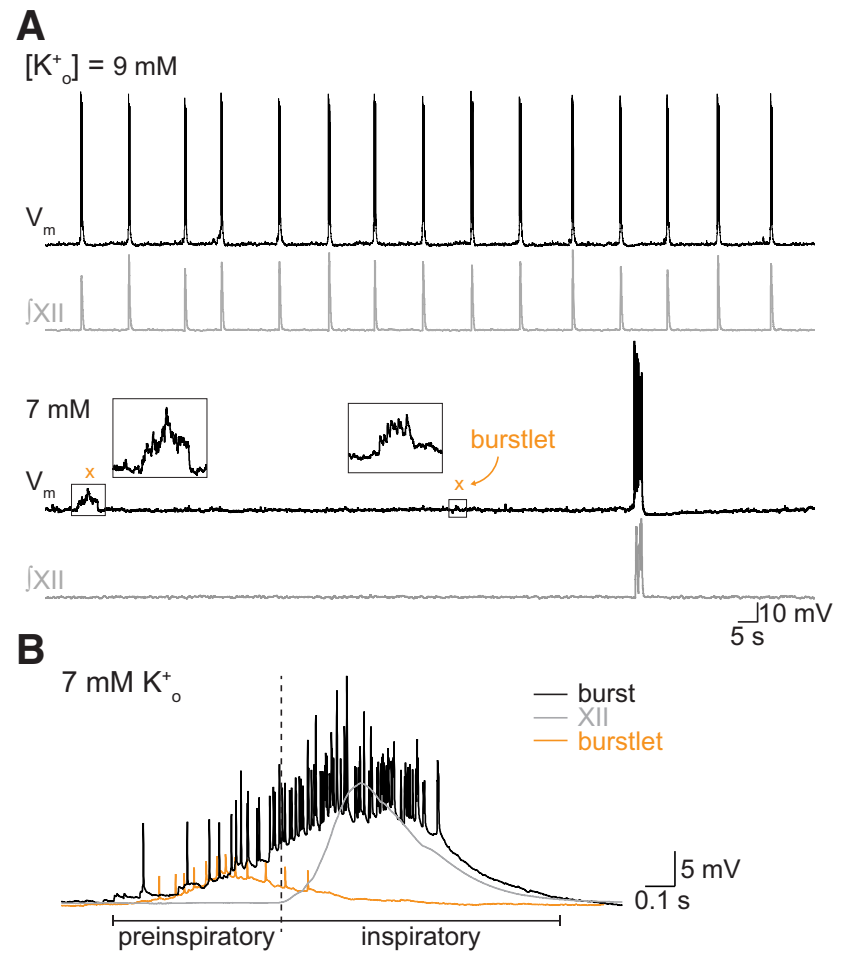

Figure 5. Properties of burstlets recorded intracellularly. $\boldsymbol{A}$, Whole-cell recording $\left(\mathrm{V}_{\mathrm{m}}\right.$, top traces) from an inspiratory preBötC neuron with XII output (gray, lower traces) at 9 and $7 \mathrm{mM}$ external $\mathrm{K}^{+}$concentration, i.e., $\left[\mathrm{K}^{+}\right]_{0}$. Insets magnify burstlets at $7 \mathrm{mM}\left[\mathrm{K}^{+}\right]_{0}$. B, Cycle-triggered averages of burstlets (orange) and inspiratory bursts (black) recorded intracellularly with XII output (gray). Preinspiratory and inspiratory burst phases are marked. $\boldsymbol{A}, \boldsymbol{B}$ have separate voltage and time calibrations.

(Fig. $6 A, B$ ). We predicted that if $89 \%$ of inspiratory neurons participate in burstlets, then the frequency of composite rhythm obtained in whole-cell recordings should be comparable to that obtained in field recordings. We found no difference in $\mathrm{f}_{\text {preBötc }}$ measured via whole-cell and field recordings at 7 or $9 \mathrm{mM}\left[\mathrm{K}^{+}\right]_{0}$. However, at $6 \mathrm{mM}\left[\mathrm{K}^{+}\right]_{\text {, }}$, $f_{\text {preBötc }}$ was significantly lower in whole-cell recordings compared to field recordings (Fig. 6A,B; Table 4). Additionally, at all $\left[\mathrm{K}^{+}\right]_{\mathrm{o}}$ levels, the $\mathrm{f}_{\mathrm{XII}}$ calculated in whole-cell recordings and field recordings remain the same. These data imply that relatively fewer inspiratory preBötC neurons are burstlet-active at $6 \mathrm{mM}\left[\mathrm{K}^{+}\right]_{\text {。 compared to }} 7$ or 9 $\mathrm{mM}$. Simultaneous triple recordings of $D b \times 1$-derived preBötC rhythmogenic neurons, preBötC population activity, and XII motor output demonstrate that individual neurons do not participate in every population burstlet (Fig. 6C).

\section{Burstlets occur in subsets of inspiratory preBötC neurons}

To investigate how many and which neurons participate in burstlets, we recorded inspiratory $D b \times 1$-derived preBötC neurons in Dbx1;Ai148 slices while simultaneously monitoring XII motor output (Figs. 7, 8). We recorded three to nine imaging planes per preBötC with $12 \pm 7$ active neurons per plane (range 3-27) for an average of $62 \pm 20$ inspiratory neurons recorded per Dbx1;Ai148 slice. Then, we manipulated $\left[\mathrm{K}^{+}\right]_{0}$ to examine burst $(9 \mathrm{mM} ; n=6$ 


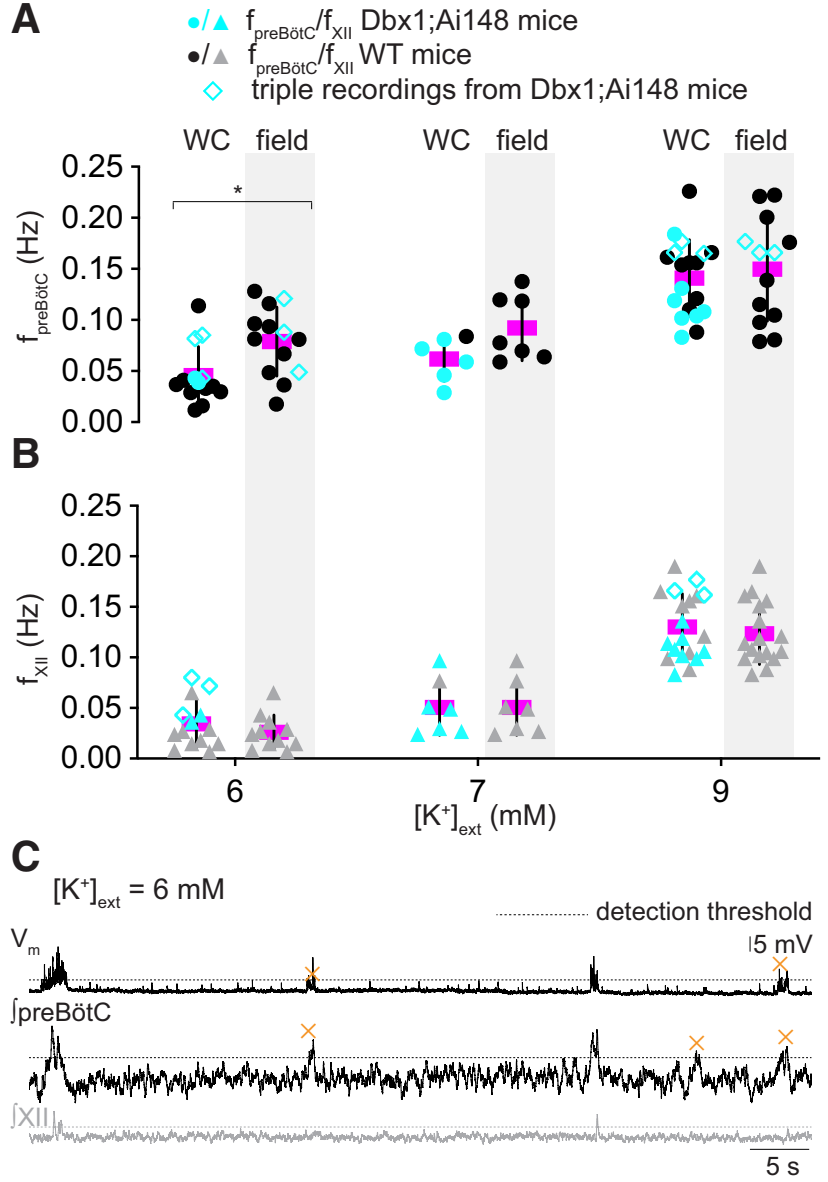

Figure 6. preBötC inspiratory neurons do not participate in every burstlet. $\boldsymbol{A}, \boldsymbol{B}, \mathrm{f}_{\text {preBötc }}$ (filled circles) and $\mathrm{f}_{\mathrm{XII}}$ (filled triangles) measured during separate or simultaneous whole-cell and field recordings at three levels of external $\mathrm{K}^{+}$concentration, i.e., $\left[\mathrm{K}^{+}\right]_{0}: 6,7$, and $9 \mathrm{mM}$. At each $\left[\mathrm{K}^{+}\right]_{0}$ level, whole-cell $(\mathrm{WC})$ is at left and the preBötC field recording is at right (with light gray background shading). Cyan symbols represent measurements from $D b x 1$-derived preBötC neurons in Dbx1;Ai148 reporter mice. Cyan unfilled diamonds represent measurements from simultaneous triple recording of $D b \times 1$-derived preBötC rhythmogenic neuron, preBötC population activity, and XII motor output. The frequency from each slice preparation is shown with mean frequency (magenta) and SD (vertical lines). Asterisk indicates statistical significance at $p<0.05$. C. Whole-cell recording $\left(\mathrm{V}_{\mathrm{m}}\right.$, top trace) from a $D b \times 1$-derived inspiratory preBötC neuron with preBötC field recording ( $\int$ preBötC, middle trace) and XII ( $\int \mathrm{XII}$, bottom trace) at $6 \mathrm{mM} \mathrm{K}^{+}$. Dashed lines mark the $95 \% \mathrm{Cl}$. Orange $\times$ symbols indicate burstlets. Vertical calibration only applies to the top trace $\left(V_{m}\right)$. Time calibration applies to all traces.

slices) and burstlet $(7 \mathrm{mM}, n=6$ slices; $6 \mathrm{mM}, n=2$ slices) rhythms.

At $9 \mathrm{mM}\left[\mathrm{K}^{+}\right]_{\mathrm{o}}, 20 \pm 9 \%$ of the $D b \times 1$-derived inspiratory neurons were active during burstlets (Fig. 7). Additionally, a neuron that was active during one burstlet was not always active during other burstlets (Fig. 8A, neurons 2, 3, 9 , 14). On changing to $7 \mathrm{mM}\left[\mathrm{K}^{+}\right]_{\mathrm{o}}, 17 \pm 15 \%$ of the Dbx1-derived inspiratory neurons were active during burstlets (Fig. 7). Again, a neuron that participated in one
Table 4. Comparison of frequencies of preBötC composite rhythm measured in whole-cell recordings and field recordings at 6,7 , and $9 \mathrm{mM}\left[\mathrm{K}^{+}\right]_{\text {o }}$

\begin{tabular}{llcl}
\hline$\left[\mathrm{K}^{+}\right]_{\mathrm{o}}(\mathrm{mM})$ & Whole cell $(\mathrm{Hz})$ & Field $(\mathrm{Hz})$ & $p$ value \\
6 & $0.04 \pm 0.03$ & $0.08 \pm 0.03$ & 0.04 \\
7 & $0.06 \pm 0.02$ & $0.09 \pm 0.03$ & 0.21 \\
9 & $0.14 \pm 0.04$ & $0.14 \pm 0.06$ & 0.65
\end{tabular}

Frequencies are reported as mean $\pm \mathrm{SD}$. The reported $p$ values (calculated using Welch's $t$ test) compare samples of composite frequency measured from the preBötC in either whole-cell or field recordings.

burstlet did not always participate in the next burstlet (Fig. $8 B$, neurons $2,3,9)$. On changing to $6 \mathrm{mM}\left[\mathrm{K}^{+}\right]_{\mathrm{o}}, 23 \%$ of Dbx 1 -derived neurons were active during burstlets (Fig. 7). These data show that the subset of $D b \times 1$-derived preBötC neurons that participates in burstlets constitutes $17-23 \%$ of the population active during inspiratory bursts and that the composition of the burstlet-active subset varies from cycle to cycle.

\section{Discussion}

Inspiratory breathing movements emanate from neural activity in the preBötC but its rhythmogenic mechanisms remain incompletely understood and, some might argue, misunderstood. There appears to be a dichotomy between the mechanisms underlying rhythmogenesis and those governing motor pattern. Here, we investigate this rhythm-pattern dichotomy to unravel the neural mechanisms of preBötC functionality.

\section{Defunct theories of inspiratory rhythmogenesis and why burstlets are viable explanation}

Theories of rhythmogenesis fall into three camps. The first posits a ring of mutually inhibitory neurons that generates sequential phases of the breathing cycle including preBötC inspiratory bursts (Richter, 1982; Smith et al., 2007, 2013; Ausborn et al., 2018). The second theoretical framework emphasizes bursting-pacemaker neurons; the synchronization of pacemakers serves as a template for network activity (Feldman and Cleland, 1982; Johnson et al., 1994; Rekling and Feldman, 1998; Butera et al.,

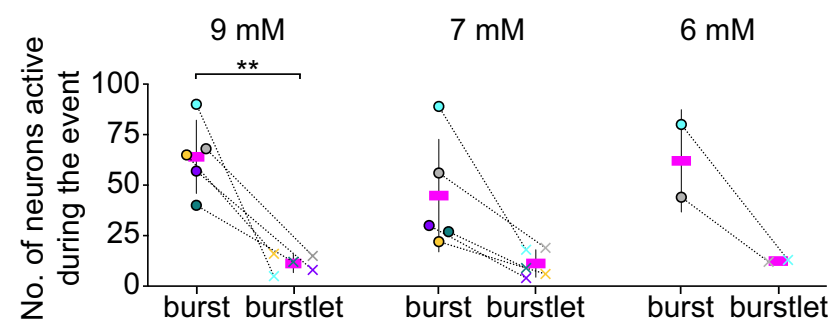

Figure 7. Burstlets occur in subset of preBötC inspiratory neurons. Group data showing the number of neurons active during bursts and burstlets from Dbx1;Ai148 slices at 9, 7, and $6 \mathrm{mM}$ external $\mathrm{K}^{+}$concentration, i.e., $\left[\mathrm{K}^{+}\right]_{\mathrm{o}}$. Active neuron counts are illustrated for each slice preparation for bursts (filled circles) and burstlets ( $\times$ symbols). The dataset is color coded for each slice. Only two slices were sufficiently rhythmically active at $6 \mathrm{mM}$ $\left[\mathrm{K}^{+}\right]_{\mathrm{o}}$ to obtain reliable measurements of burstlets within the 2 -min recording duration of imaging time series. Asterisks signify statistical significance at $p<0.01$. 
A

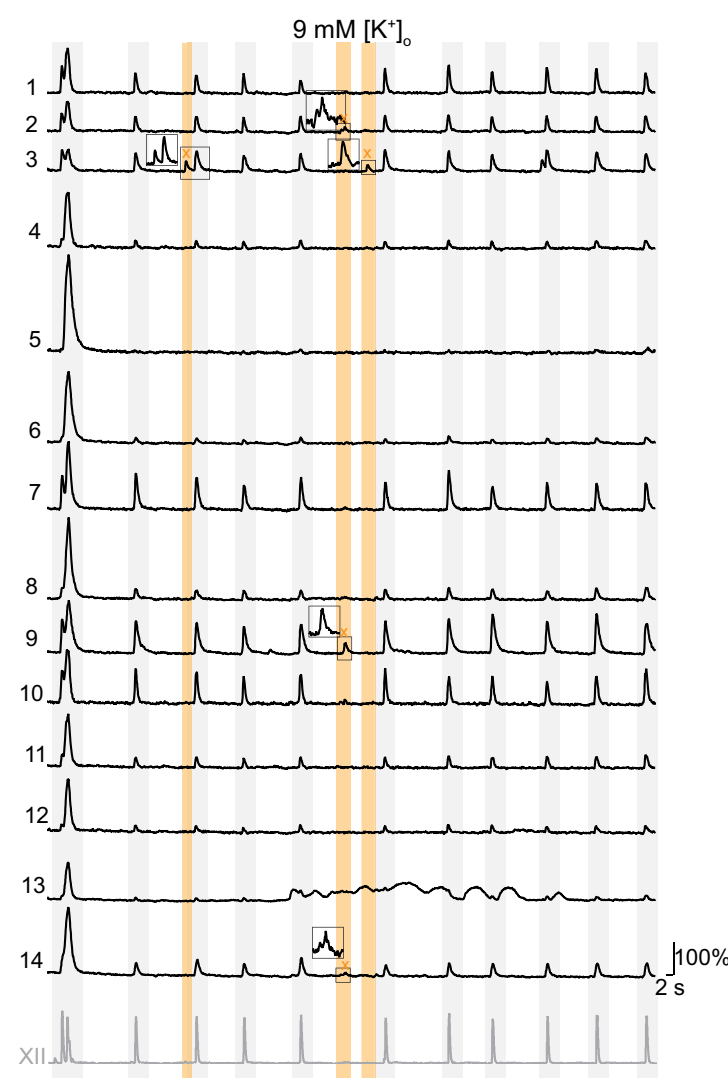

B

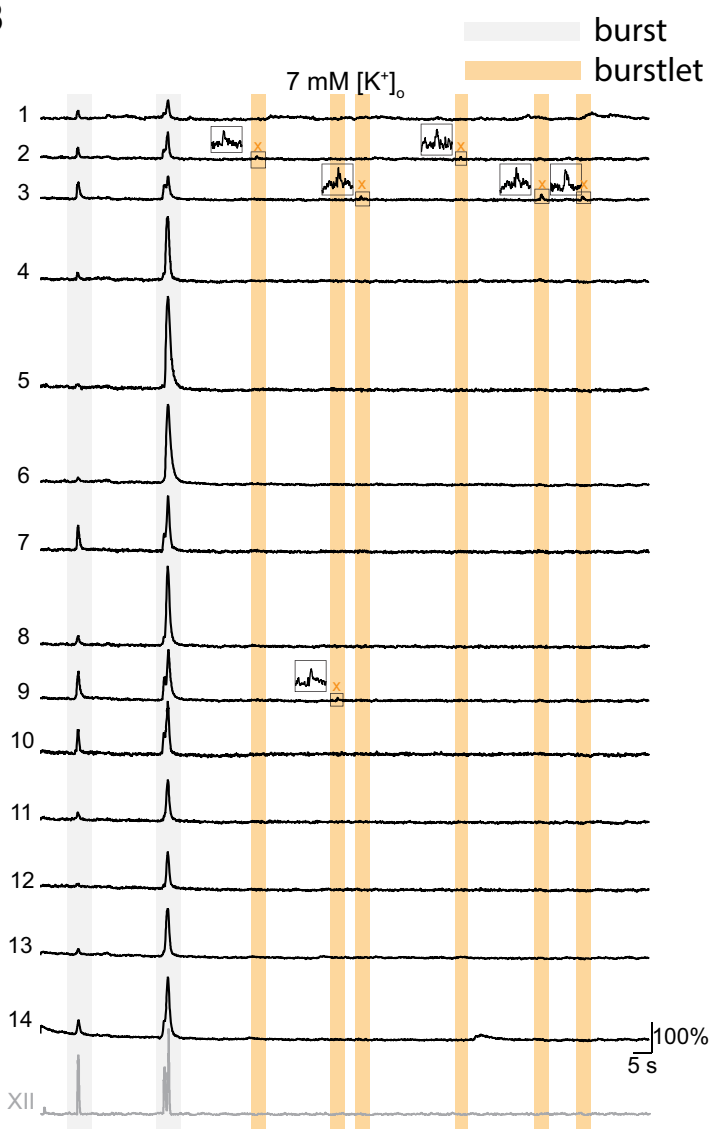

Figure 8. Burstlets occur in dynamic subsets of preBötC inspiratory neurons. Bursts and burstlets in a group of 14 Dbx1-derived preBötC neurons in a typical Dbx1;Ai148 mouse slice preparation with inspiratory XII output (lowest traces, gray). Inspiratory cycles are background shaded in light gray. Burstlet cycles are background shaded in light orange. $\boldsymbol{A}, \boldsymbol{B}$ show population activity in 9 and $7 \mathrm{mM}$ external $\mathrm{K}^{+}$concentration, i.e., $\left[\mathrm{K}^{+}\right]_{\mathrm{o}}$, respectively. Insets in $\boldsymbol{A}, \boldsymbol{B}$ show select burstlets.

1999a,b; Ramirez et al., 2004). The third theory, dubbed a group pacemaker, posits that recurrent synaptic activity triggers mixed-cationic conductances to produce inspiratory bursts (Rekling et al., 1996; Rekling and Feldman, 1998; Del Negro and Hayes, 2008; Rubin et al., 2009).

Disinhibition of the preBötC (Shao and Feldman, 1997; Brockhaus and Ballanyi, 1998; Janczewski et al., 2013; Sherman et al., 2015; Marchenko et al., 2016; Cregg et al., 2017; Baertsch et al., 2018), as well as attenuation of pacemaker conductances (Del Negro et al., 2002, 2005; Peña et al., 2004; Pace et al., 2007; Koizumi and Smith, 2008) and mixed-cationic conductances (Koizumi et al., 2018; Picardo et al., 2019) neither perturbs the frequency in the predicted manner nor stops breathing in vivo or inspiratory rhythms in vitro, which falsifies all three rhythmogenic mechanisms. Nevertheless, the key to understanding rhythmogenesis may be found in what these theories get wrong: inextricable neural bursts that culminate the inspiratory phase of the cycle.

To consider the iconoclastic notion of rhythmogenesis in the absence of bursts we, like Kam and Feldman (Kam et al., 2013a; Feldman and Kam, 2015), focus on the preinspiratory phase that ordinarily leads to bursts and motor output. The preinspiratory phase is a hallmark of rhythmogenesis, marking early-activating rhythmogenic interneurons (Onimaru et al., 1987, 1988; Smith et al., 1990; Rekling et al., 1996; Carroll and Ramirez, 2013; Carroll et al., 2013). Concurrent excitation of four to nine preBötC interneurons in vitro, by photolytic glutamate uncaging (Smith et al., 1990; Rekling et al., 1996; Kam et al., 2013b; Sun et al., 2019), can effectively trigger a preBötC network burst after a latency of $100-400 \mathrm{~ms}$, similar to the duration of the preinspiratory phase. There are two important take-aways: first, small numbers $(<10)$ of coactive neurons can trigger a burst; second, the burst occurs after sufficient time for percolation of network interactions to reach threshold. Kam and Feldman (Kam et al., 2013a) divorced preinspiratory activity from bursts, showing that rhythmic burstlets remained in their absence, and argued that burstlets represent the rhythmogenic substrate.

We also observed preBötC field activity like burstlets absent XII output at all $\left[\mathrm{K}^{+}\right]_{\mathrm{o}}$ levels. Manipulating network excitability detaches the preinspiratory and inspiratory components of preBötC burst and affects their prevalence. Field and whole-cell recordings from preBötC neurons showed that burstlet rise time, duration, and amplitude match preinspiratory activity. These data affirm the hypothesis that both burstlets and preinspiratory activity share a common rhythmogenic mechanism. 


\section{How many constituent neurons activate during bursts and burstlets?}

The frequency of preBötC composite rhythm differed significantly between field recordings and whole-cell recordings at $6 \mathrm{mM}\left[\mathrm{K}^{+}\right]_{\mathrm{O}}$. Field recordings reflect activity among many preBötC neurons, whereas whole-cell recordings reflect just one constituent preBötC neuron. Two things change as excitability decreases. First, fewer neurons participate in burstlets but field recordings still detect the collective events. Second, any neuron singled out for whole-cell recording is less likely to be part of the burstletactive subpopulation. The initial burstlet report showed that $\sim 89 \%$ of preBötC neurons active during bursts also participate in burstlets. Here, using photonics to monitor $\sim 62$ Dbx1-derived inspiratory burst-active neurons, we found $\sim 20 \%$ participate in burstlets. We conclude using our whole-cell recordings and photonic recordings that the subset of burstlet-active neurons is inconstant and appears lower than $89 \%$.

The estimated size of the rhythmogenic population is 560-650 preBötC neurons (Hayes et al., 2012; Wang et al., 2014) so the burstlet-active subpopulation numbers between $112-130(20 \%)$ and 500-580 (89\%). That seemingly large range can explain why burstlet amplitude varies with $\left[\mathrm{K}^{+}\right]_{0}$ : increasing excitability can recruit potentially hundreds of additional constituent preBötC neurons to the burstlet-active subpopulation. Whether or not the fraction of burstlet-active preBötC neurons is closer to $20 \%$ or $89 \%$, very few $(<10)$ coactive preBötC neurons can trigger full bursts and motor output (Kam et al., 2013b; Sun et al., 2019) so the relative fraction of burstletactive neurons may not be a critical parameter governing network activity.

\section{Burstlet mechanism: network oscillator that depends on recurrent synaptic excitation}

Here, $f_{\text {preBötc }}$ increased monotonically with $\left[\mathrm{K}^{+}\right]_{0}$. Because $\left[\mathrm{K}^{+}\right]_{0}$ modulates network excitability via direct influence on baseline membrane potential in preBötC constituent neurons, we conclude that preBötC composite rhythm is voltage dependent. In contrast, the initial burstlet report showed no statistically significant disparity between $\mathrm{f}_{\text {preBötc }}$ at 6 vs $9 \mathrm{mM}\left[\mathrm{K}^{+}\right]_{0}$, yet there was a disparity for $3 \mathrm{mM}$ versus either 6 or $9 \mathrm{mM}$. This left the question open as to whether burstlet rhythm might be some form of synchronized voltage-independent biochemical oscillator in constituent neurons. Monotonically increasing $f_{\text {preBötc }}$ as a function of $\left[\mathrm{K}^{+}\right]_{0}$ rules out that possibility. A significant difference in $f_{\text {preBötC }}$ and $f_{X I I}$, at 3 and $4 \mathrm{mM}\left[\mathrm{K}^{+}\right]_{\mathrm{o}}$, suggests that burstlets maintain the preBötC rhythm. These data imply that burstlets are rhythmogenic in nature, but that concept of rhythmogenicity has not been proven and awaits definitive testing.

So, what mechanism does give rise to burstlets? Our whole-cell recordings show temporal summation of EPSPs during burstlets. We held membrane potential at -60 $\mathrm{mV}$, which imposes steady-state deactivation of the persistent $\mathrm{Na}^{+}$current (Del Negro et al., 2002; Ptak et al., 2005; Yamanishi et al., 2018). Therefore, burstlets do not reflect voltage-dependent bursting properties and do appear to reflect recurrent synaptic excitation.

In general, network oscillators (distinct from pacemaker or inhibition-based models) rely on recurrent excitation among constituent rhythmogenic neurons (Grillner, 2006; Grillner and El Manira, 2020). Modifying the neuronal excitability influences the relative fraction of spontaneously active neurons in the network, and, for silent neurons, the proximity of baseline membrane potential to spike threshold. Increasing excitability therefore magnifies the number of neurons interacting, facilitates synaptic drive summation, and accelerates the process of recurrent excitation to directly influence frequency. We conclude that burstlets are not only rhythmogenic, but also follow dynamics of recurrent excitation, i.e., a network oscillator model of rhythmogenesis.

\section{Frequency control differs for sighs compared to burstlets and bursts}

The preBötC can generate burstlets, inspiratory bursts, and sigh-related bursts (Lieske et al., 2000; Ruangkittisakul et al., 2008). Here, $\mathrm{f}_{\text {sigh }}$ increased as the $\left[\mathrm{K}^{+}\right]_{\mathrm{o}}$ level increased, which at face value suggests voltage dependence (akin to $f_{\text {preBötC }}$ and $f_{X I I}$ as argued above). However, $f_{\text {sigh }}$ is an order of magnitude lower than $f_{\text {preBötC }}$ and $f_{X I I}$. Further, the $\left[\mathrm{K}^{+}\right]_{\mathrm{o}}$-dependent increase in $\mathrm{f}_{\text {sigh }}$ is 20 times less steep than that of $f_{\text {preBötc }}$ or $f_{X I I}$. These observations suggest that the mechanism for frequency control of the composite preBötC rhythm and the XII motor output do not similarly apply to sigh rhythm. We propose that sigh frequency control is not voltage dependent like burstlets and inspiratory bursts, which implicates a biochemical oscillator for sigh rhythms that interacts with the network oscillator underlying burstlets and inspiratory bursts to bring about the less-steep $\mathrm{f}_{\text {sigh }}$ versus $\left[\mathrm{K}^{+}\right]_{\text {o }}$ curve. We cannot yet specify how the sigh rhythm is generated; it is beyond the scope of this paper.

\section{Pattern generation}

Burstlet amplitude depends on the excitability too. Above, we inferred that additional constituent neurons (perhaps $\sim 100 \mathrm{~s})$ are recruited as excitability increases. In contrast, the amplitude of preBötC bursts and XII motor output are not voltage dependent across $\left[\mathrm{K}^{+}\right]_{\mathrm{o}}$ levels. Although burstlet amplitude changes with excitability, the rise time, fall time, and duration of burstlet events do not vary with $\left[\mathrm{K}^{+}\right]_{\mathrm{o}}$ levels. First, we conclude that the network dynamics that recruit burstlet-active neurons, and govern the temporal evolution of burstlets, are largely the same at different levels of excitability. Second, we conclude that preBötC activity, once passing threshold, triggers a cascade that activates all (or nearly all) preBötC neurons, and also activates premotor and motor neurons. That cascade probably depends on synaptic connectivity among pattern-related preBötC neurons and premotor neurons outside of the preBötC, but not preBötC excitability per se.

We cannot yet specify how activity during the preinspiratory phase reaches a threshold for burst generation. It may have to do with a quorum: a certain number of rhythmogenic interneurons must be active (i.e., spiking) to trigger an irreversible cascade that ostensibly activates all 
(or nearly all) preBötC neurons. Or, it may have to do with synchrony: a certain number of rhythmogenic interneurons must be spiking in sync to trigger that cascade (Ashhad and Feldman, 2019). The former focuses on mass action of constituent interneurons wherein temporal precision is inconsequential. The latter focuses on phasic precision rather than mass action. Our present data cannot distinguish which mechanism is at work but given that burstlet amplitude is voltage-dependent and the number of constituent neurons participating in burstlets may vary at any given level of excitability, the quorum model seems less feasible.

There is an existing framework for understanding both rhythm and pattern generation of the preBötC. Dbx1derived preBötC neurons are inspiratory rhythmogenic (Bouvier et al., 2010; Gray et al., 2010; Wang et al., 2014; Cui et al., 2016; Koizumi et al., 2016; Vann et al., 2016, 2018; Baertsch et al., 2018), playing key preinspiratory and burst-generating roles (Picardo et al., 2013; Cui et al., 2016), and some serving exclusively premotor function (Revill et al., 2015). It may be possible to identify subsets of predominantly rhythmogenic versus predominantly premotor or pattern-related Dbx1-derived preBötC neurons based on intrinsic membrane properties (Picardo et al., 2013) or neuropeptide somatostatin expression (Cui et al., 2016). Those two classification schemes are not mutually exclusive because many somatostatin- and somatostatin receptor-expressing preBötC neurons are Dbx1-derived (Gray et al., 2010). It is also possible that particular ion channels serve in a pattern-related capacity within core rhythmogenic neurons. For example, transient receptor potential (Trp) channels in Dbx1-derived preBötC neurons whose activation amplifies inspiratory burst magnitude (Koizumi et al., 2018; Picardo et al., 2019) also maintain the tidal volume of inspiratory breaths in unanesthetized adult mice (Picardo et al., 2019).

Our results affirm the ideas presented in the burstlet hypothesis (Kam et al., 2013a) that rhythm and pattern generation are discrete processes, which nevertheless both begin in the preBötC. Burstlets, subthreshold from the standpoint of motor discharge, appear to reflect the core rhythmogenic mechanism involving recurrent synaptic excitation.

\section{References}

Ashhad S, Feldman JL (2019) Network synchronization and synchrony propagation: emergent elements of inspiration. BioRxiv 664946.

Ausborn J, Koizumi H, Barnett WH, John TT, Zhang R, Molkov YI, Smith JC, Rybak IA (2018) Organization of the core respiratory network: insights from optogenetic and modeling studies. PLoS Comput Biol 14:e1006148.

Baertsch NA, Baertsch HC, Ramirez JM (2018) The interdependence of excitation and inhibition for the control of dynamic breathing rhythms. Nat Commun 9:843.

Bielle F, Griveau A, Narboux-Nême N, Vigneau S, Sigrist M, Arber S, Wassef M, Pierani A (2005) Multiple origins of Cajal-Retzius cells at the borders of the developing pallium. Nat Neurosci 8:1002-1012.

Borrus DS, Conradi Smith GD, Del Negro CA (2019) Role of synaptic inhibition in the coupling of the respiratory rhythms that underlie eupnea and sigh behaviors. BioRxiv 721829.
Bouvier J, Thoby-Brisson M, Renier N, Dubreuil V, Ericson J, Champagnat J, Pierani A, Chédotal A, Fortin G (2010) Hindbrain interneurons and axon guidance signaling critical for breathing. Nat Neurosci 13:1066-1074.

Brockhaus J, Ballanyi K (1998) Synaptic inhibition in the isolated respiratory network of neonatal rats. Eur J Neurosci 10:38233839.

Butera RJ, Rinzel J, Smith JC (1999a) Models of respiratory rhythm generation in the pre-Bötzinger complex. I. Bursting pacemaker neurons. J Neurophysiol 82:382-397.

Butera RJ, Rinzel J, Smith JC (1999b) Models of respiratory rhythm generation in the pre-Bötzinger complex. II. Populations of coupled pacemaker neurons. J Neurophysiol 82:398-415.

Carroll MS, Ramirez JM (2013) Cycle-by-cycle assembly of respiratory network activity is dynamic and stochastic. J Neurophysiol 109:296-305.

Carroll MS, Viemari JC, Ramirez JM (2013) Patterns of inspiratory phase-dependent activity in the in vitro respiratory network. J Neurophysiol 109:285-295.

Cregg JM, Chu KA, Dick TE, Landmesser LT, Silver J (2017) Phasic inhibition as a mechanism for generation of rapid respiratory rhythms. Proc Natl Acad Sci USA 114:12815-12820.

Cui Y, Kam K, Sherman D, Janczewski WA, Zheng Y, Feldman JL (2016) Defining preBötzinger complex rhythm- and patterngenerating neural microcircuits in vivo. Neuron 91:602-614.

Daigle TL, Madisen L, Hage TA, Valley MT, Knoblich U, Larsen RS, Takeno MM, Huang L, Gu H, Larsen R (2018) A suite of transgenic driver and reporter mouse lines with enhanced brain cell type targeting and functionality. Cell 174:465-480.

Del Negro CA, Hayes JA (2008) A 'group pacemaker' mechanism for respiratory rhythm generation. J Physiol 586:2245-2246.

Del Negro CA, Koshiya N, Butera RJ, Smith JC (2002) Persistent sodium current, membrane properties and bursting behavior of pre-Bötzinger complex inspiratory neurons in vitro. J Neurophysiol 88:2242-2250.

Del Negro CA, Morgado-Valle C, Hayes JA, Mackay DD, Pace RW, Crowder EA, Feldman JL (2005) Sodium and calcium currentmediated pacemaker neurons and respiratory rhythm generation. J Neurosci 25:446-453.

Del Negro CA, Kam K, Hayes JA, Feldman JL (2009) Asymmetric control of inspiratory and expiratory phases by excitability in the respiratory network of neonatal mice in vitro. J Physiol 587:12171231.

Del Negro CA, Funk GD, Feldman JL (2018) Breathing matters. Nat Rev Neurosci 19:351-367.

Feldman JL, Cleland CL (1982) Possible roles of pacemaker neurons in mammalian respiratory rhythmogenesis. Cell Pacemak 2:101119.

Feldman JL, Kam K (2015) Facing the challenge of mammalian neural microcircuits: taking a few breaths may help. J Physiol 593:3-23.

Funk GD, Greer JJ (2013) The rhythmic, transverse medullary slice preparation in respiratory neurobiology: contributions and caveats. Respir Physiol Neurobiol 186:236-253.

Gray PA, Hayes JA, Ling GY, Llona I, Tupal S, Picardo MCD, Ross SE, Hirata T, Corbin JG, Eugenin J, Del Negro CA (2010) Developmental origin of preBötzinger complex respiratory neurons. J Neurosci 30:14883-14895.

Grillner S (2006) Biological pattern generation: the cellular and computational logic of networks in motion. Neuron 52:751-766.

Grillner S, El Manira A (2020) Current principles of motor control, with special reference to vertebrate locomotion. Physiol Rev 100:271320.

Hayes JA, Wang X, Del Negro CA (2012) Cumulative lesioning of respiratory interneurons disrupts and precludes motor rhythms in vitro. Proc Natl Acad Sci USA 109:8286-8291.

Janczewski WA, Tashima A, Hsu P, Cui Y, Feldman JL (2013) Role of inhibition in respiratory pattern generation. J Neurosci 33:54545465. 
Johnson SM, Smith JC, Funk GD, Feldman JL (1994) Pacemaker behavior of respiratory neurons in medullary slices from neonatal rat. J Neurophysiol 72:2598-2608.

Johnson SM, Koshiya N, Smith JC (2001) Isolation of the kernel for respiratory rhythm generation in a novel preparation: the preBötzinger complex "island." J Neurophysiol 85:1772-1776.

Kam K, Worrell JW, Janczewski WA, Cui Y, Feldman JL (2013a) Distinct inspiratory rhythm and pattern generating mechanisms in the preBötzinger complex. J Neurosci 33:9235-9245.

Kam K, Worrell JW, Ventalon C, Emiliani V, Feldman JL (2013b) Emergence of population bursts from simultaneous activation of small subsets of preBötzinger complex inspiratory neurons. J Neurosci 33:3332-3338.

Koizumi H, Smith JC (2008) Persistent $\mathrm{Na}^{+}$and $\mathrm{K}^{+}$-dominated leak currents contribute to respiratory rhythm generation in the preBötzinger complex in vitro. J Neurosci 28:1773-1785.

Koizumi H, Mosher B, Tariq MF, Zhang R, Koshiya N, Smith JC (2016) Voltage-dependent rhythmogenic property of respiratory preBötzinger complex glutamatergic, Dbx1-derived, and somatostatinexpressing neuron populations revealed by graded optogenetic inhibition. eNeuro 3:ENEURO.0081-16.2016.

Koizumi H, John TT, Chia JX, Tariq MF, Phillips RS, Mosher B, Chen Y, Thompson R, Zhang R, Koshiya N, Smith JC (2018) Transient receptor potential channels TRPM4 and TRPC3 critically contribute to respiratory motor pattern formation but not rhythmogenesis in rodent brainstem circuits. eNeuro 5:ENEURO.0332-17.2018.

Lieske SP, Thoby-Brisson M, Telgkamp P, Ramirez JM (2000) Reconfiguration of the neural network controlling multiple breathing patterns: eupnea, sighs and gasps. Nat Neurosci 3:600-607.

Marchenko V, Koizumi H, Mosher B, Koshiya N, Tariq MF, Bezdudnaya TG, Zhang R, Molkov YI, Rybak IA, Smith JC (2016) Perturbations of respiratory rhythm and pattern by disrupting synaptic inhibition within pre-Bötzinger and Bötzinger complexes. eNeuro 3:ENEURO.0011-16.2016.

National Research Council (2011) Guide for the care and use of laboratory animals, Ed 8. Washington, DC: National Academies Press.

Onimaru H, Arata A, Homma I (1987) Localization of respiratory rhythm-generating neurons in the medulla of brainstem-spinal cord preparations from newborn rats. Neurosci. Lett 78:151-155.

Onimaru H, Arata A, Homma I (1988) Primary respiratory rhythm generator in the medulla of brainstem-spinal cord preparation from newborn rat. Brain Res 445:314-324.

Pace RW, Mackay DD, Feldman JL, Del Negro CA (2007) Role of persistent sodium current in mouse preBötzinger complex neurons and respiratory rhythm generation. J Physiol 580:485-496.

Peña F, Parkis MA, Tryba AK, Ramirez J-M (2004) Differential contribution of pacemaker properties to the generation of respiratory rhythms during normoxia and hypoxia. Neuron 43:105-117.

Picardo MCD, Weragalaarachchi KT, Akins VT, Del Negro CA (2013) Physiological and morphological properties of Dbx1-derived respiratory neurons in the pre-Bötzinger complex of neonatal mice. $J$ Physiol 591:2687-2703.

Picardo MCD, Sugimura YK, Dorst KE, Kallurkar PS, Akins VT, Ma X, Teruyama R, Guinamard R, Kam K, Saha MS, Del Negro CA (2019) Trpm4 ion channels in pre-Bötzinger complex interneurons are essential for breathing motor pattern but not rhythm. PLoS Biol 17:e2006094.

Ptak K, Zummo GG, Alheid GF, Tkatch T, Surmeier DJ, McCrimmon DR (2005) Sodium currents in medullary neurons isolated from the pre-Bötzinger complex region. J Neurosci 25:5159-5170.

Ramirez JM, Tryba AK, Peña F (2004) Pacemaker neurons and neuronal networks: an integrative view. Curr Opin Neurobiol 14: 665-674.

Rekling JC, Feldman JL (1998) PreBötzinger complex and pacemkaer neurons: hypothesized site and kernel for respiratory rhythm generation. Annu Rev Physiol 60:385-405.

Rekling JC, Champagnat J, Denavit-Saubié M (1996) Electroresponsive properties and membrane potential trajectories of three types of inspiratory neurons in the newborn mouse brain stem in vitro. $J$ Neurophysiol 75:795-810.

Revill AL, Vann NC, Akins VT, Kottick A, Gray PA, Del Negro CA, Funk GD (2015) Dbx1 precursor cells are a source of inspiratory XII premotoneurons. Elife 4:e12301.

Richter DW (1982) Generation and maintenance of the respiratory rhythm. J Exp Biol 100:93-107.

Ruangkittisakul A, Schwarzacher SW, Secchia L, Ma Y, Bobocea N, Poon BY, Funk GD, Ballanyi K (2008) Generation of eupnea and sighs by a spatiochemically organized inspiratory network. J Neurosci 28:2447-2458.

Ruangkittisakul A, Panaitescu B, Ballanyi K (2011) $\mathrm{K}^{+}$and $\mathrm{Ca}^{2+}$ dependence of inspiratory-related rhythm in novel "calibrated" mouse brainstem slices. Respir Physiol Neurobiol 175:37-48.

Ruangkittisakul A, Kottick A, Picardo MCD, Ballanyi K, Del Negro CA (2014) Identification of the pre-Bötzinger complex inspiratory center in calibrated "sandwich" slices from newborn mice with fluorescent Dbx1 interneurons. Physiol Rep 2:e12111.

Rubin JE, Hayes JA, Mendenhall JL, Del Negro CA (2009) Calciumactivated nonspecific cation current and synaptic depression promote network-dependent burst oscillations. Proc Natl Acad Sci USA 106:2939-2944.

Schindelin J, Arganda-Carreras I, Frise E, Kaynig V, Longair M, Pietzsch T, Preibisch S, Rueden C, Saalfeld S, Schmid B, Tinevez JY, White DJ, Hartenstein V, Eliceiri K, Tomancak P, Cardona A (2012) Fiji: an open-source platform for biological-image analysis. Nat Methods 9:676-682.

Schneider CA, Rasband WS, Eliceiri KW (2012) NIH Image to ImageJ: 25 years of image analysis. Nat Methods 9:671-675.

Shao XM, Feldman JL (1997) Respiratory rhythm generation and synaptic inhibition of expiratory neurons in pre-Bötzinger complex: differential roles of glycinergic and GABAergic neural transmission. J Neurophysiol 77:1853-1860.

Sherman D, Worrell JW, Cui Y, Feldman JL (2015) Optogenetic perturbation of preBötzinger complex inhibitory neurons modulates respiratory pattern. Nat Neurosci 18:408-414.

Smith JC, Greer JJ, Liu GS, Feldman JL (1990) Neural mechanisms generating respiratory pattern in mammalian brain stem-spinal cord in vitro. I. Spatiotemporal patterns of motor and medullary neuron activity. J Neurophysiol 64:1149-1169.

Smith JC, Ellenberger HH, Ballanyi K, Richter DW, Feldman JL (1991) Pre-Bötzinger complex: a brainstem region that may generate respiratory rhythm in mammals. Science 254:726-729.

Smith JC, Abdala APL, Koizumi H, Rybak IA, Paton JFR (2007) Spatial and functional architecture of the mammalian brain stem respiratory network: a hierarchy of three oscillatory mechanisms. J Neurophysiol 98:3370-3387.

Smith JC, Abdala AP, Borgmann A, Rybak IA, Paton JF (2013) Brainstem respiratory networks: building blocks and microcircuits. Trends Neurosci 36:152-162.

Sun X, Thörn Pérez C, Nagaraj Halemani D, Shao XM, Greenwood M, Heath S, Feldman JL, Kam K (2019) Inspiratory rhythmogenic activity is burst-independent and opioid-sensitive. BioRxiv 665034.

Vann NC, Pham FD, Hayes JA, Kottick A, Del Negro CA (2016) Transient suppression of Dbx1 preBötzinger interneurons disrupts breathing in adult mice. PLoS One 11:e0162418.

Vann NC, Pham FD, Dorst KE, Del Negro CA (2018) Dbx1 preBötzinger complex interneurons comprise the core inspiratory oscillator for breathing in unanesthetized adult mice. eNeuro 5.

Wang X, Hayes JA, Revill AL, Song H, Kottick A, Vann NC, LaMar MD, Picardo MCD, Akins VT, Funk GD, Del Negro CA (2014) Laser ablation of Dbx1 neurons in the pre-Bötzinger complex stops inspiratory rhythm and impairs output in neonatal mice. Elife 3:e03427.

Yamanishi T, Koizumi H, Navarro MA, Milescu LS, Smith JC (2018) Kinetic properties of persistent $\mathrm{Na}^{+}$current orchestrate oscillatory bursting in respiratory neurons. J Gen Physiol 150:1523-1540. 